\title{
Serum Fatty Acid Synthase as a Marker of Esophageal Neoplasia
}

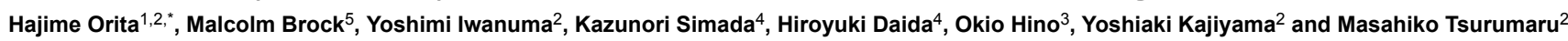

${ }^{1}$ Department of Surgery, Juntendo Shizuoka hospital, Shizuoka, Shizuoka, Japan

${ }^{2}$ Department of Esophageal and Gastroenterological Surgery, Juntendo University School of Medicine, Tokyo, Japan

${ }^{3}$ Department of Pathology, Juntendo University School of Medicine, Tokyo, Japan, Tokyo, Japan

${ }^{4}$ Department of Cardiology, Juntendo University School of Medicine, Tokyo, Japan, Tokyo, Japan

${ }^{5}$ Department of Surgery, Johns Hopkins School of Medicine, Baltimore, USA

*Corresponding author: Hajime Orita, Department of Surgery, Juntendo Shizuoka Hospital, Shizuoka, Japan, Tel: 055-948-3111; Fax: 055-946-0514; E-mail: oriori@juntendo.ac.jp

Rec date: Jan 8, 2016; Acc date: Feb 8; 2016; Pub date: Feb 11, 2016

Copyright: ( 2016 Orita $\mathrm{H}$, et al. This is an open-access article distributed under the terms of the Creative Commons Attribution License, which permits unrestricted use, distribution, and reproduction in any medium, provided the original author and source are credited.

\begin{abstract}
Background: Fatty Acid Synthase (FAS) is highly expressed in many human cancers. Previously, we reported that FAS is expressed at very high levels in esophageal cancer, and that the growth of these cancers can be inhibited by pharmacological agents that target this enzyme. We also have reported that this enzyme is overexpressed not only in tissue, but also in serum in patients with various cancers. In this present analysis, we investigate the serum level of this enzyme in patients, and consider whether FAS can be a prognositc tumor marker or one of early detection.
\end{abstract}

Materials and Methods: By using an ELISA kit, we measured the serum levels of FAS in 154 patients, who underwent surgical resection of esophageal cancer at the Juntendo Hospital in Tokyo, Japan. We also measured the serum FAS levels of 153 healthy volunteers recruited as normal controls from a cardiology research group.

Finally, we considered how FAS levels correlated with pathological and clinical data.

Results: Serum FAS levels in esophageal cancer patients were significantly higher than in healthy control subjects(13.2 ug/ml vs. $2.3 \mathrm{ul} / \mathrm{ml}$, respectively). FAS levels did not correlate with clinical or-pathological data.

Conclusions: Serum FAS levels are expressed at significantly high levels in human esophageal carcinomas. FAS serum levels may be a tumor marker candidate for early esophageal cancer detection.

Keywords: Fatty acid synthase; Esophageal cancer; ELISA Kit

\section{Introduction}

Recently, in the clinical setting, molecularly-targeted agents, such as EGFR, VGFR and Her-2, are attracting lots of attention and showing good performance in chemotherapy, especially for breast and colorectal cancers [1]. But they have efficacious limitations, because tumor cells are heterogenous, especially esophageal and gastric cancers, with continous inflammation [2].

The transformation of malignant cells from normal cells is accompanied by dysregulation of metabolic pathways [3]. It is wellknown that anaerobic metabolism is accelerated in many cancer cells [4]. Fatty acid synthesis (FAS) is one of the reasons for this accelerated metabolic prosess $[5,6]$.

FAS, the enzyme responsible for fatty acid biosynthesis, is overexpressed in many human cancers, and is reported to be correlated with cancer growth and poor prognosis and On one hand, inhibition of FAS results in decreased cell proliferation and, loss of cell viability. [4-6].

FASN is this Protein Coding gene. Diseases that have been reported to associate with FASN include trophoblastic neoplasm and obesity.
Among their related pathways are MAPK signaling pathway and Proteoglycans in cancer.

On the other hand, many reports indicate that FAS is also critical for supporting energy production, because newly synthesized fatty acids are rapidly incorporated into neutral- and phospho-lipid stores [7].

Recently, Nomura and Cravatt F [8,9] carefully reviewed the literature and found that lipogenesis may contribute to cancer by building and regulating cell membranes, not energy resource. Though it remains unclear, metabolic therapy may be appropriate as a nextgeneration anti-tumor treatment $[5,6,10,11]$.

We already have reported that FAS is expressed at very high levels in esophageal cancer (both squamous and adenocarcinoma histologies), and high expression is also seen in Barrett's esophagus with dysplasia [12].

Esophageal cancer is the eighth leading cause of cancer death and one of the most clinically aggressive of all solid cancers [13-15]. Although there are two histologic variants of esophageal cancer, squamous and adenocarcinoma, both with distinctive epidemiologic patterns, the major risk factors (smoking, dietary factors) and many clinical features are similar between both histologies. More than $60 \%$ 
of esophageal cancersare diagnosed as advanced stage tumors. Despite new diagnostic and therapeutic approaches, esophageal cancer still has a poor prognosis, with 5-year survival rates between $10-13 \%$ [16]. Recently, it has been reported that an inherited deficiency in the enzyme aldehyde dehydrogenase 2 (ALDH2) is strongly correlated with the presence of esophageal cancer [17,18]. Measuring this enzyme during screening has led to a recommendation for increased GI fiber consumption for those at a high risk of esophageal cancer [14,15]. Moreover, esophageal adenocarcinomas are common in western countries, primarily due to the high incidence of Gastro-Esophageal Reflex Disease (GERD).

Although FAS is found to be overexpressed in many solid tumors, its role in esophageal cancer has not been extensively evaluated. We have reported that it seems to be up-regulated during the early stages of tumorigenesis [12].

The aim of this study is to evaluate FAS as a marker of esophageal cancer by using an ELISA assay to measure FAS serum levels in patients with esophageal cancer, and in those without cancer.

\section{Materials and Methods}

\section{Serum samples}

A total of 307 serum samples were analyzed. Fasting preoperative serum samples, numbering 154 in total, were obtained from patients undergoing a three field lymph node dissection esophagectomy at the Juntendo University Hospital. Of these, 29 patients received neoadjuvant chemoradiaton therapy and all patients had squamous cell carcinoma. We analysed 79/154 patients with the tumor marker data and compared them with the FAS serum data. Serum samples were also obtained from 153 healthy volunteers from Dr. Shimada who leads the Juntendo University's Department of Cardiology. All controls were matched to study patients by age and BMI, and underwent a physical exam to ensure that they had no malignant disease. The mean age of the study group was 63.6 years old (range, 46-80 years) and 63.9 years old for the healthy control group (range, 33-83 years). All of the samples were collected with the approval of the Juntendo Committee for Clinical Investigation.

\section{FAS ELISA}

A total of $100 \mu \mathrm{L}$ of serum was analyzed with a commercially available ELISA kit, FAS-detect ELISA (FASgen, Baltimore, MD), according to the manufacturer's recommendations.

Briefly, sera were incubated in a 96 -well capture plate ona plate shaker for $90 \mathrm{~min}$ at room temperature. The plate was then washed five times with wash buffer. FAS enzyme conjugate was added and the plate was incubated for $60 \mathrm{~min}$, and the wash was repeated. Serum FAS levels werevisualized by color change upon addition of tetramethylbenzidinesubstrate followed by the addition of a substrate stopsolution. Absorbance values were read at $450 \mathrm{~nm}$ using a SpectraMax spectrophotometer. FAS concentrations were determined by interpolation from the standard curve.

\section{Statistical analysis}

Statistical analysis was generated using the Graph Pad Prism 5.0 (GraphPad Software, San Diego, CA). For univariate analysis, Student $\mathrm{T}$ test and Mann-Whitney (nonparametric test) were done using the same software statistical program. Differences were considered significant at $\mathrm{p}<0.05$. Receiver operating characteristics (ROC) analysis was used to evaluate the diagnostic value of FAS to identify the optimal cut off values. Sensitivity and specificity, positive and negative predictive values of FAS were profiled using curves.

\section{Results}

Serum FAS Levels were not correlated with serum T-cholesterol or Triglyceride (TG) levels

Serum FAS levels were analyzed in 154 patients with esophageal cancer and in 153 healthy controls matched by age, and BMI. There was no difference in serum T-cholesterol and triglyceride levels between groups. This suggests that serum FAS levels were not associated with total body adipose (Table 1).

\begin{tabular}{|l|l|l|l|}
\hline & Control & $\begin{array}{l}\text { Esophageal } \\
\text { cancer } \\
\text { patients }\end{array}$ & P-value \\
\hline age & $\begin{array}{l}33 \sim 83 \\
(\text { mean } 63.9)\end{array}$ & $\begin{array}{l}46 \sim 80 \\
\text { (mean 63.6) }\end{array}$ & \\
\hline Sex (man: women) & $115: 38(3: 1)$ & $134: 20(6: 1)$ & 0.757 \\
\hline BMI & 24.11 & 21.13 & 0.279 \\
\hline T-cho & 197.09 & 189.01 & 0.181 \\
\hline TG & 157.29 & 108.69 & \\
\hline
\end{tabular}

Table 1: Shows clinicopathological features. Control groups and esophageal cancer patients are not significantly different in BMI, serum T-cho, and TG levels (Student T test).

Serum FAS levels in esophageal cancer patients are significantly higher than in healthy volunteers.

The median and first quartile FAS levels in patients with esophageal cancer were $13.22 \mathrm{ng} / \mathrm{mL}$ and $10.25 \mathrm{ng} / \mathrm{mL}$, respectively. For healthy controls, they were $2.32 \mathrm{ng} / \mathrm{mL}$ and $5.10 \mathrm{ng} / \mathrm{mL}$, respectively (Figure 1). FAS levels were therefore significantly higher in patients with esophageal cancer than in healthy controls ( $\mathrm{p}<0.05$, Student's $t$ test) (Table 2). FAS scores did not seem to differ with progressive clinical stage (Table 2).

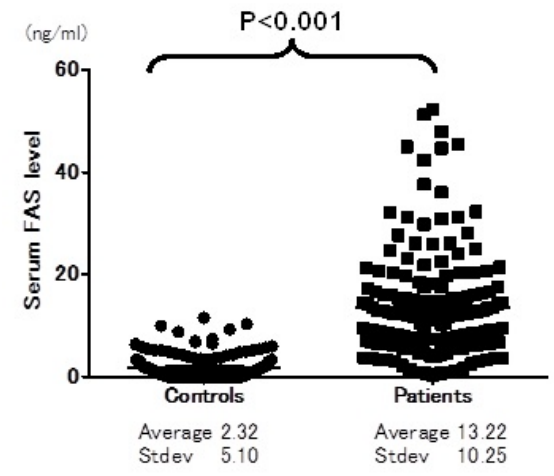

Figure 1: Esophageal cancer patients' serum FAS levels were significantly higher than in the control group (Student T-test). 


\begin{tabular}{|c|c|c|c|c|}
\hline & & Patients & FAS score & $\mathrm{P}$-value \\
\hline \multirow{6}{*}{ Stage } & 0 & 4 & 10.53 & \multirow{6}{*}{$\mathrm{P}=0.486$} \\
\hline & 1 & 12 & 10.72 & \\
\hline & ॥ & 37 & 13.61 & \\
\hline & III & 58 & 13.74 & \\
\hline & IVa & 36 & 12.18 & \\
\hline & Ivb & 7 & 12.21 & \\
\hline \multirow{5}{*}{ T } & T1a & 4 & 10.53 & \multirow{5}{*}{$\mathrm{P}=0.363$} \\
\hline & T1b & 29 & 11.75 & \\
\hline & T2 & 25 & 16.19 & \\
\hline & T3 & 82 & 13.06 & \\
\hline & $\mathrm{T} 4$ & 14 & 12.61 & \\
\hline \multirow{2}{*}{$\mathbf{N}$} & $(+)$ & 114 & 14.09 & \multirow{2}{*}{$P=0.212$} \\
\hline & $(-)$ & 40 & 10.73 & \\
\hline
\end{tabular}

Table 2: Univarate analysis- Serum FAS levels did not correlate with stage, $\mathrm{T}$ or lymph node factors (Mann-Whitney (nonparametric test)).

A comparison of serum FAS levels with known tumor markers revealed that the marker, CyFRA, is most closely correlated with serum FAS (Table 3).

\begin{tabular}{|l|l|l|l|l|}
\hline & & 79 cases & FAS level & P- value \\
\hline \multirow{3}{*}{ CEA } & $<5$ (negative) & 67 & 16.46 & \multirow{2}{*}{$\mathrm{P}=0.893$} \\
\cline { 2 - 4 } & $\geq 5$ (positive) & 12 & 16.06 & \\
\hline \multirow{2}{*}{ SCC } & $<2$ (negative) & 68 & 13.92 & \multirow{2}{*}{$\mathrm{P}=0.185$} \\
\cline { 2 - 4 } & $\geq 2$ (positive) & 11 & 21.08 & \\
\hline \multirow{2}{*}{ CA 19-9 } & $<37$ (negative) & 77 & 15.43 & \multirow{2}{*}{$\mathrm{P}=0.581$} \\
\cline { 2 - 4 } & $\geq 37$ (positive) & 2 & 11.71 & \\
\hline \multirow{2}{*}{ CyFRA } & $<2$ (negative) & 41 & 13.87 & \multirow{2}{*}{$\mathrm{P}=0.005$} \\
\cline { 2 - 4 } & $\geq 2$ (positive) & 38 & 17.03 & \\
\hline
\end{tabular}

Table 3: A comparison of FAS levels with known tumor markers.

(Mann-Whitney (nonparametric test))

A comparison of serum FAS levels with known tumor markers revealed that the marker,

CyFRA, is most closely correlated with serum FAS.

\section{Diagnostic accuracy of serum FAS in esophageal cancer patients}

To evaluate the diagnostic value of serum FAS levels, we used the ROC method to calculate the sensitivity and specificity of FAS. Using the ROC curve, we found that 126 of 154 patients with esophageal cancer had an elevated FAS level (sensitivity 84.8\%) and 145 of 153 healthy control patients did not have elevated FAS levels (specificity, $89.5 \%$ ) (Figure 2).

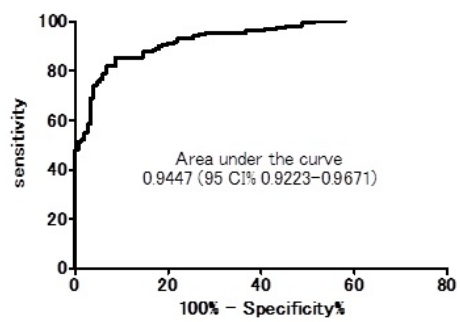

Figure 2: ROC curves of serum FAS, the AUC in progressive disease was 0.9447

Positive serum FAS levels found in.84\% of Patients Negative for Four Known Tumor Biomarkers

In 32 patients (32/79) who were negative for four existing tumor markers, 27 patients had serum FAS levels that were more than 5 $\mathrm{ng} / \mathrm{ml}$. According to our ROC curve therefore, at a defined cut off level of $5 \mathrm{ng} / \mathrm{ml}, 84 \%$ of these biomarker negative patients could be diagnosed with esophageal cancer (Table 4). Thus, in our dataset, serum FAS can diagnose more than 55 patients who otherwise had no known positive tumor biomarkers that are currently in use in the clinic.

\begin{tabular}{|l|l|l|l|}
\hline & & \multicolumn{2}{|l|}{ All Four tumor marker } \\
\hline & & Positive & Negative \\
\hline \multirow{3}{*}{ FAS } & negative & 2 & 5 \\
\cline { 2 - 4 } & positive & 45 & 27 \\
\hline
\end{tabular}

Table 4: ROC analysis.

$84 \%$ of these biomarker negative patients could be diagnosed with esophageal cancer at a defined cut off level of $5 \mathrm{ng} / \mathrm{ml}$.

\section{Discussion}

Tumor markers for early detection of esophageal cancer are urgently needed to improve the prognosis of patients with this highly aggressive disease. Even when patients begin to manifest symptoms such as dysphagia, congestion, or hoarseness, they are already at an advanced stage or too late for surgical intervention. Development of a noninvasive diagnostic test could facilitate screening of high-risk individuals, such as heavy alcohol drinkers and smokers.

Identification of serum protein markers of esophageal cancer could help provide such a noninvasive diagnostic screening tool. Elevated FAS protein levels have been detected as early biomarkers in the serum of patients with pancreatic cancer patients [13].

In this study, we investigated the role of serum FAS in esophageal cancer. We show that there is no relationship between FAS levels, Tcholesterol, and TG in patients. This suggests that, in cancer cells, FAS is very important for novel synthesis of fatty acid regardless of the patient's weight, food intake or other factors. This result confirms our hypothesis that FAS is a key target for cancer therapy. 
Cancer metabolism is now being increasingly studied globally [19-21]. There are 2 pathways that are used by cancer cells to generate energy, the glycolytic system and the fatty acid synthesis pathway. The glycolytic system is commonly exploited for PET scanning in the clinic. But PET scanning is imperfect; it especially cannot detect tumors in diabetic patients. We already have reported a good correlation between PET scanning and FAS metabolism [20]. PET scanning also incurs a high cost. So, as an alternative to PET, it is important to note that FAS up-regulation occurs early in tumorigenesis. Our previous report suggested that cells with Barrett's dysplasia have high levels of FAS. FAS may also be at a high level during carcinogenesis, so malignant cells necessarily use large amounts of energy. Thus, continuous inhibition of FAS could be protective against carcinogenesis.

In the future, we plan to measure serum FAS levels postoperatively in order to detect cancer recurrence and/or metastasis. According to our ROC data, serum FAS levels are highly specify, but have low sensitivity. This suggests that FAS could be a marker for the presence of malignancy, but not an independent marker of esophageal cancer.

\section{Conclusion}

Serum FAS levels are expressed at significantly high levels in human esophageal carcinoma. It may be a putative tumor marker.

\section{Acknowledgments}

The authors would like to express their deepest appreciation to Professors Dr. Edward Gabrielson in Department of Pathology, providing direction about this FAS project, constructive comments, and warm encouragement.

\section{Conflicts of Interest}

MEXT-Supported Program for the Strategic Research Foundation at Private Universities 2015-2020

\section{References}

1. Kalia M (2015) Biomarkers for personalized oncology: recent advances and future challenges. Metabolism 64: S16-S21.

2. Nagaraja V, Eslick GD (2015) HER2 expression in gastric and oesophageal cancer: a meta-analytic review. J Gastrointest Oncol 6: 143-154.

3. DeBerardinis RJ (2008) Is cancer a disease of abnormal cellular metabolism? New angles on an old idea. Genet Med 10: 767-777.

4. Krause HP, Probst H, Schneider F (1971) Cell division and energy dependent metabolic power of Ehrlich-ascites-tumor cells under aerobic and anaerobic conditions. Chemie Biochemie Biophysik Biologie 26: 780-788.
5. Kuhajda FP (2000) Fatty-acid synthase and human cancer: new perspectives on its role in tumor biology. Nutrition 16: 202-208.

6. Kuhajda FP, Jenner K, Wood FD, Hennigar RA, Jacobs LB, et al. (1994) Fatty acid synthesis: a potential selective target for antineoplastic therapy. Proceedings of the National Academy of Sciences of the United States of America 91: 6379-6383.

7. Menendez JA, Lupu R (2007) Fatty acid synthase and the lipogenic phenotype in cancer pathogenesis. Nature reviews Cancer 7: 763-777.

8. Nomura DK, Long JZ, Niessen S, Hoover HS, Ng SW, et al. (2010) Monoacylglycerol lipase regulates a fatty acid network that promotes cancer pathogenesis. Cell 140: 49-61.

9. Nomura DK, Cravatt BF (2013) Lipid metabolism in cancer. Biochimica et biophysica acta 1831: 1497-1498.

10. Epstein JI, Carmichael M, Partin AW (1995) OA-519 (fatty acid synthase) as an independent predictor of pathologic state in adenocarcinoma of the prostate. Urology 45: 81-86.

11. Pizer ES, Jackisch C, Wood FD, Pasternack GR, Davidson NE, et al. (1996) Inhibition of fatty acid synthesis induces programmed cell death in human breast cancer cells. Cancer Res56: 2745-2747.

12. Orita H, Coulter J, Tully E, Abe M, Montgomery E, et al. (2010) High levels of fatty acid synthase expression in esophageal cancers represent a potential target for therapy. Cancer Biol Ther 10: 549-554.

13. Walter K, et al. (2009) Serum fatty acid synthase as a marker of pancreatic neoplasia. Cancer Epidemiol Biomarkers Prev 18: 2380-2385.

14. Hori H, Kawano T, Endo M, Yuasa Y (1997) Genetic polymorphisms of tobacco- and alcohol-related metabolizing enzymes and human esophageal squamous cell carcinoma susceptibility. J Clin Gastroenterol 25: 568-575.

15. Matsuo K, Hamajima N, Shinoda M, Hatooka S, Inoue M, et al. (2001) Gene-environment interaction between an aldehyde dehydrogenase-2 (ALDH2) polymorphism and alcohol consumption for the risk of esophageal cancer. Carcinogenesis 22(6): pp: 913-916.

16. Ye T, Sun Y, Zhang Y, Chen H (2013) Three-field or two-field resection for thoracic esophageal cancer: a meta-analysis. The Annals of thoracic surgery 96: 1933-1941.

17. Yokoyama A, Muramatsu T, Ohmori T, Higuchi S, Hayashida M, et al. (1996) Esophageal cancer and aldehyde dehydrogenase-2 genotypes in Japanese males. Cancer Epidemiol Biomarkers Prev 5: 99-102.

18. Brooks PJ, Enoch M, Goldman D, Li T, Yokoyama A (2009) The alcohol flushing response: an unrecognized risk factor for esophageal cancer from alcohol consumption. PLoS medicine 6: e50.

19. Leung K (2004) [11C]Acetate, in Molecular Imaging and Contrast Agent Database (MICAD) Bethesda.

20. Lee JS, Orita H, Gabrielson K, Alvey S, Hagemann RL, et al. (2007) FDGPET for pharmacodynamic assessment of the fatty acid synthase inhibitor C75 in an experimental model of lung cancer. Pharmaceutical research 24: 1202-1207.

21. Vavere AL, Kridel SJ, Wheeler FB, Lewis JS (2008) 1-11C-acetate as a PET radiopharmaceutical for imaging fatty acid synthase expression in prostate cancer. Journal of nuclear medicine 49: 327-334. 